\title{
Radiographic analysis of the restoration of hip joint center following open reduction and internal fixation of acetabular fractures: a retrospective cohort study
}

\author{
Hong-fei Shi, Jin Xiong, Yi-xin Chen", Jun-fei Wang and Yin-he Wang
}

\begin{abstract}
Background: Unfavorable reduction is considered one of the key factors leading to joint degeneration and compromised clinical outcome in acetabular fracture patients. Besides the columns, walls, and superior dome, the postoperative position of hip joint center (HJC), which is reported to affect hip biomechanics, should be considered during the assessment of quality of reduction. We aimed to evaluate the radiographic restoration of HJC in acetabular fractures treated with open reduction and internal fixation.

Methods: Patients with a displaced acetabular fracture that received open reduction and internal fixation in the authors' institution during the past five years were identified from the trauma database. The horizontal and vertical shifts of HJC were measured in the standard anteroposterior view radiographs taken postoperatively. The radiographic quality of fracture reduction was graded according to Matta's criteria. The relationships between the shift of HJC and the other variables were evaluated.

Results: Totally 127 patients with 56 elementary and 71 associated-type acetabular fractures were included, wherein the majority showed a medial (89.0\%) and proximal (93.7\%) shift of HJC postoperatively. An average of $2.8 \mathrm{~mm}$ horizontal and $2.2 \mathrm{~mm}$ vertical shift of HJC were observed, which correlated significantly with the quality of fracture reduction $(P<0.001$ for both). The horizontal shift of HJC correlated with the fracture type $(P=0.022)$.

Conclusions: The restoration of HJC correlates with the quality of reduction in acetabular fractures following open reduction and internal fixation. Further studies are required to address the effects of HJC shift on the biomechanical changes and clinical outcomes of hip joint, especially in poorly reduced acetabular fractures.
\end{abstract}

Keywords: Acetabular fracture, Open reduction and internal fixation, Hip joint center, Radiography

\section{Background}

Acetabular fracture remains as a major challenge to orthopaedic surgeons despite of decades of improvement in its operative management. Following well-planned open reduction and internal fixation (ORIF), a good to excellent result can be estimated in a large part of the patients with acetabular fractures. Meanwhile, the complication rate is still high, which leads to poor long-term outcomes in approximately $20 \%$ of the patients $[1,2]$.

\footnotetext{
* Correspondence: dr.chenyixin@gmail.com Department of Orthopaedics, Nanjing Drum Tower Hospital, The Affiliated
Hospital of Nanjing University Medical School, No. 321 Zhongshan Road, Department of Orthopaedics, Nanjing Drum Tower Hospital, The Affiliated
Hospital of Nanjing University Medical School, No. 321 Zhongshan Road, Nanjing, China
}

(C) 2014 Shi et al.; licensee BioMed Central Ltd. This is an Open Access article distributed under the terms of the Creative Commons Attribution License (http://creativecommons.org/licenses/by/2.0), which permits unrestricted use, distribution, and reproduction in any medium, provided the original work is properly credited.
Post-traumatic osteoarthritis, usually accompanied with loss of hip motion and increase of pain, has been considered one of the most common complications associated with compromised outcomes in acetabular fractures [3]. It's generally accepted that biomechanical alterations in hip joint, caused by an unfavorable fracture reduction, play undoubtable roles in the development of arthritis. In previous studies, special emphases were placed to analyze the changes of intraarticular contact characteristics and the loss of stability after acetabular fractures $[4,5]$.

The hip joint center (HJC), also known as the rotation center of hip joint, is considered crucial for the biomechanical reconstruction of the hip joint during total 
hip arthroplasty (THA) and revision surgeries [6,7]. When an acetabular fracture occurs, it's not rare that the position of HJC will change following the destruction of acetabulum and innominate bone. Since an unfavorable position of HJC was reported to cause increased hip load, compromised soft tissue balancing, and even gait changes [8,9], it might contribute to the development of post-traumatic arthritis in patients with acetabular fractures as well. Currently, the postoperative assessment of fracture reduction focuses on the residual displacement of columns, walls, and the superior dome [10,11]. A clearer understanding of the restoration of postoperative HJC in acetabular fractures, which was merely addressed previously, might shed lights on further optimization of the surgical management. In this study, we aimed to quantify the postoperative shift of HJC radiographically, and to evaluate the relationship between the shift of HJC and the quality of fracture reduction following ORIF of acetabular fractures.

\section{Methods}

We retrospectively reviewed the patients with acetabular fractures that were recorded in the trauma database in the authors' department. The patients were admitted through emergency department or referred from other hospitals. Totally 201 displaced fractures (193 patients) were considered not fitted for Matta's criteria of nonoperative treatment [12], and then received ORIF between January 2007 and December 2011. Of these reviewed cases, we included those with a full series of standard radiographs, including pre- and postoperative anteroposterior (AP), iliac oblique and obturator oblique Judet views, as well as preoperative computed tomography (CT) scan of the pelvis. Patients with bilateral acetabular fracture, associated fractures of ipsilateral femoral head, fracture of pelvic ring, or those operated on more than two weeks after injury were excluded. The study protocol was approved by the Medical Ethics Committee of Nanjing Drum Tower Hospital (Ref. No. 113217). The study had adhered to the STROBE guidelines for observational studies.

Following thorough preoperative evaluations, all the patients were operated on by two of the senior attendings (JX and YXC). Surgical approaches including Kocher-Langenbeck, ilioinguinal, combined or extensile approaches were determined by the fracture pattern to facilitate reduction and fixation of the innominate bone and the articular surface of acetabulum (Table 1). Definitive fixation was applied with reconstructive plates and screws (Synthes, Switzerland) to stabilize the fracture according to the standard techniques recommended by Letournel [13].

Radiographic examination was performed right after the removal of drainage (usually 48 to 72 hours) postoperatively. Standard AP radiograph of the pelvis were taken with the patients placed supine and their feet in a standard position to minimize the effect of rotation of the hip joint. To evaluate the restoration of the HJC following ORIF, we measured the vertical and horizontal shifts of the postoperative center of femoral head from the estimated center of femoral head referring to the contralateral intact hip joint (Figure 1). In brief, the vertical axis of the pelvis (VA line) was defined by connecting the middle of the inter-sacroiliac line and the middle of the pubic symphysis in digitized postoperative AP view radiographs. With built-in tools, the distance (D1) between the postoperative femoral head center and the VA line was measured using Digimizer image analysis software (MedCalc Software Ltd, Mariakerke, Belgium), the same as the distance (D2) between the contralateral intact femoral head center and the VA line. The horizontal shift $(\mathrm{X})$ of the postoperative $\mathrm{HJC}$ was then calculated as the absolute value of the difference between D1 and D2 $(\mathrm{X}=|\mathrm{D} 1-\mathrm{D} 2|)$. The vertical shift $(\mathrm{Y})$ was measured as the distance between the paralleled D1 line and D2 line (Figure 1). The direction of the horizontal and vertical shift was also recorded. All the measurements were calibrated with the diameters of the $3.5 \mathrm{~mm}$ cortical screws measured in digitized radiographs as reference. Two senior orthopaedic surgeons (JFW and WJW) performed the measurements independently, with the interobserver quantitative data averaged for statistical analysis. The interobserver reliability was examined via interclass correlation coefficient (ICC).

The quality of fracture reduction was evaluated by measuring the residual displacement of the columns, walls, and superior dome in digitized anteroposterior (AP) and two oblique (iliac oblique and obturator oblique) Judet view radiographs [12]. The maximum residual displacement (MRD) was used to grade the quality of reduction according to Matta's criteria: anatomical (MRD less than 2 millimeters), imperfect (MRD between 2 and $3 \mathrm{~mm}$ ), poor (MRD more than $3 \mathrm{~mm}$ ), and secondary congruence (articular congruence of the acetabulum whilst displacement of the innominate bone in both-column fractures).

Statistical analysis was performed using IBM SPSS version 19.0 software (SPSS Inc., Chicago, IL), with statistical significance set at a $P$ value of less than 0.05 . The quantitative data of length of surgery and shift of HJC (X and Y) were demonstrated as mean $\pm \mathrm{SD}$ and compared among different types of fracture using one-way ANOVA. Person correlation coefficient $(r)$ or Spearman's rank correlation coefficient (rho) was conducted, as appropriate, to identify possible association between the shift of $\mathrm{HJC}$ and the other variables. Besides, the relationship of quality of fracture reduction with the other categorical factors was analyzed using Chi-square test. An a priori power analysis demonstrated that, with an effect size of 0.3 or greater, a minimum of 84 patients were required to detect a 
Table 1 Fracture types and information of surgery

\begin{tabular}{|c|c|c|c|c|c|c|}
\hline \multirow[t]{2}{*}{ Fracture type } & \multirow[t]{2}{*}{ Number } & \multirow[t]{2}{*}{ Length of surgery (min) } & \multicolumn{4}{|c|}{ Surgical approach } \\
\hline & & & Ilioinguinal & K-L & Combined & Extensile \\
\hline \multicolumn{7}{|l|}{ Elementary $(n=56)$} \\
\hline Anterior wall & 2 & $245.0 \pm 21.2$ & 2 & & & \\
\hline Anterior column & 5 & $252.0 \pm 35.5$ & 5 & & & \\
\hline Posterior wall & 31 & $106.3 \pm 21.2$ & & 31 & & \\
\hline Posterior column & 4 & $147.5 \pm 31.2$ & & 4 & & \\
\hline Transverse & 14 & $208.9 \pm 78.7$ & 3 & 9 & 2 & \\
\hline \multicolumn{7}{|l|}{ Associated $(n=71)$} \\
\hline Posterior column and posterior wall & 3 & $130.0 \pm 8.7$ & & 3 & & \\
\hline Transverse and posterior wall & 15 & $190.3 \pm 87.6$ & & 11 & 4 & \\
\hline T-shape & 17 & $265.6 \pm 93.9$ & 2 & 6 & 7 & 2 \\
\hline Anterior column and posterior hemitransverse & 12 & $278.3 \pm 62.1$ & 9 & & 3 & \\
\hline Both column & 24 & $338.5 \pm 48.4$ & 5 & & 16 & 3 \\
\hline Summary & 127 & $218.8 \pm 102.7^{\mathrm{a}, \mathrm{b}}$ & 26 & 64 & 32 & 5 \\
\hline
\end{tabular}

${ }^{a}$ significant difference among different fracture types $(P<0.05)$

${ }^{\mathrm{b}}$ significant difference among different surgical approaches $(P<0.05)$.

significant correlation between shift of HJC and quality of fracture reduction with $80 \%$ statistical power.

\section{Results}

Totally 127 patients (127 fractures) with an average age of 40.2 years (range 17 to 78 years) were included in this investigation, consisted of 82 male and 45 female patients. According to the Letournel and Judet's classification, there were 56 elementary and 71 associated-type fractures identified in preoperative radiographs and CT images (Table 1). According to our surgical records, the mean length of surgery for all the patients was 218.8 minutes (range 85 to 440 minutes), while significant difference was detected among different fracture types (one-way ANOVA, $P<0.001$ ). Comparing the use of different surgical approaches, the length of surgery also varied significantly (one-way ANOVA, $P<0.001)$.

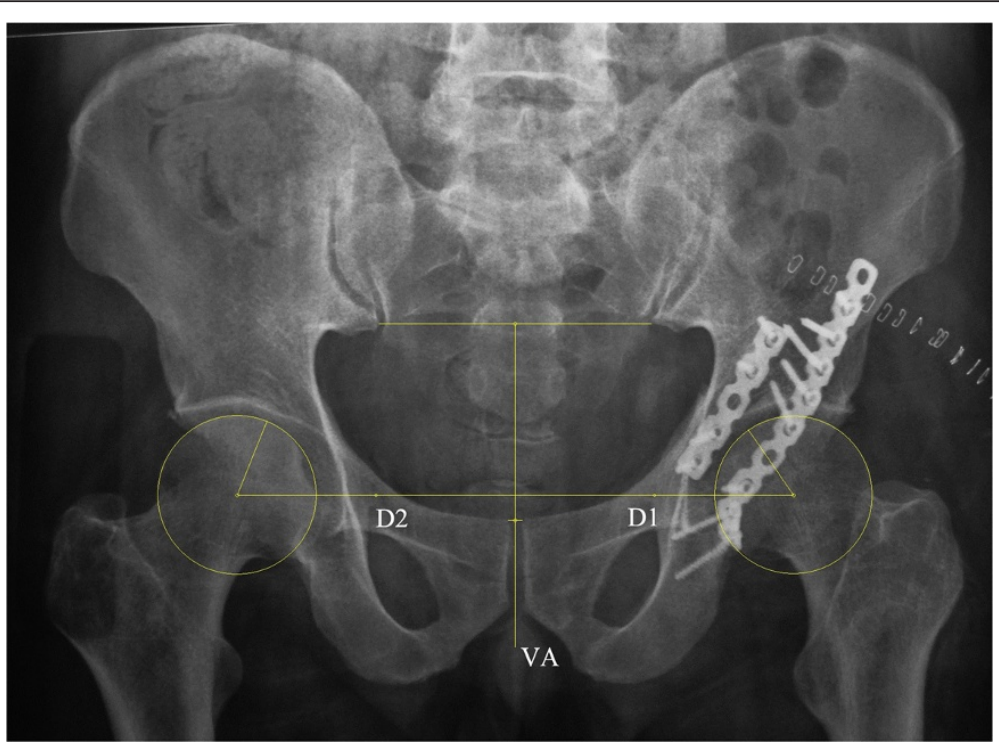

Figure 1 Radiographic measurement of the postoperative shift of hip joint center (HJC). The vertical axis of the pelvis (VA line) was defined by connecting the middle of the inter-sacroiliac line and the middle of the pubic symphysis. The distance (D1) between the postoperative femoral head center and the VA line was measured, the same as the distance (D2) between the contralateral intact femoral head center and the VA line. The horizontal shift $(X)$ of the postoperative HJC was calculated as the absolute value of the difference between D1 and D2 (X =|D1 - D2|). The vertical shift (Y) was measured as the distance between the paralleled D1 line and D2 line. 
The results of postoperative radiographic evaluation were demonstrated in Table 2. The mean horizontal and vertical shifts (X and $\mathrm{Y}$ ) of the postoperative $\mathrm{HJC}$ were $2.8 \mathrm{~mm}$ (range 0.8 to $10.7 \mathrm{~mm}$ ) and $2.2 \mathrm{~mm}$ (range 0.8 to $7.8 \mathrm{~mm}$ ) respectively, while $\mathrm{X}$ showed statistically significant correlation with the fracture type (rho $=0.204$, $P=0.022$ ). Besides, no correlation was found between the shift of HJC and the surgical approaches. A high interobserver reliability was testified with the ICC of X and $\mathrm{Y}$ was 0.88 and 0.81 respectively. Considering the direction of the shift, 113 cases (89.0\%) showed a medial shift of postoperative HJC, while 119 (93.7\%) of the vertical shift was proximal.

The quality of fracture reduction was graded radiographically as anatomical in 80 cases, imperfect in 37 cases, and poor in 10 cases (Table 2), which correlated with the type of fracture classified as elementary or associated-type (chi square $=6.689, P=0.035$ ). Both $X$ and $Y$ showed statistically significant correlation with the quality of fracture reduction (rho $=0.817$ and 0.656 respectively, $P<0.001$ for both). As shown in Figure 2, the mean $\mathrm{X}$ and $\mathrm{Y}$ were $3.5 \mathrm{~mm}$ and $2.6 \mathrm{~mm}$ respectively when an imperfect fracture reduction was achieved, which then reached $8.2 \mathrm{~mm}$ and $6.1 \mathrm{~mm}$ in poorly reduced acetabular fractures (Figure 3).

\section{Discussion}

To recover a functional and pain-free hip is the main goal in the treatment of acetabular fracture. Among the identified poor prognostic factors, unfavorable fracture reduction is considered the most important one leading to biomechanical alteration and accelerated degenerative changes in hip joint $[14,15]$. Previously, the restoration of $\mathrm{HJC}$ was merely investigated during the postoperative assessments of the quality of reduction in acetabular fractures. In this study, prior to further biomechanical investigation and clinical follow-up studies, we examined the radiographic restoration of HJC following ORIF of acetabular fracture. The results showed a $2.8 \mathrm{~mm}$ horizontal shift and a $2.2 \mathrm{~mm}$ vertical shift of postoperative HJC in average, which correlated with the radiographically graded quality of fracture reduction.

The biomechanical importance of an anatomically restored HJC has been widely investigated in THA and revision surgeries. Superior or lateral displacement of HJC, causing a decreased moment arm of abductor muscles, was testified to generate increased hip load during gait cycles and lead to higher rate of implant wear and loosening in THA [16-18]. Using mathematical models, Bicanic reported a $0.7 \%$ or $0.1 \%$ increase of hip load respectively, following every millimeter of lateral or proximal shift of HJC [9]. Similar in the opposite way, the hip load would decrease when the HJC shifted medially or distally. Considering an acetabular fracture, the alteration of the loading pattern was believed to be more complicated [4]. In our study, the majority of the cases presented varying degrees of medial and proximal shifts of HJC. It's hard, therefore, to clarify the changes of hip load caused by the shifted position of HJC in our study, unless further biomechanical studies could be conducted. However, since the mean values of the HJC shifts appeared to be relatively

Table 2 Postoperative radiographic evaluation of the shift of hip joint center (HJC) and the quality of fracture reduction

\begin{tabular}{|c|c|c|c|c|c|}
\hline \multirow[t]{2}{*}{ Fracture type } & \multicolumn{2}{|c|}{ Postoperative shift of HJC (mm) } & \multicolumn{3}{|c|}{ Quality of fracture reduction } \\
\hline & Horizontal & Vertical & Anatomical & Imperfect & Poor \\
\hline \multicolumn{6}{|l|}{ Elementary } \\
\hline Anterior wall & $2.8 \pm 1.0$ & $1.9 \pm 0.1$ & 2 & & \\
\hline Anterior column & $2.0 \pm 1.3$ & $1.5 \pm 0.5$ & 4 & 1 & \\
\hline Posterior wall & $2.0 \pm 0.7$ & $1.9 \pm 0.7$ & 26 & 5 & \\
\hline Posterior column & $2.4 \pm 1.0$ & $2.0 \pm 1.2$ & 3 & 1 & \\
\hline Transverse & $3.1 \pm 2.3$ & $2.6 \pm 1.8$ & 7 & 5 & 2 \\
\hline \multicolumn{6}{|l|}{ Associated } \\
\hline Posterior column and posterior wall & $1.7 \pm 0.5$ & $1.7 \pm 0.4$ & 3 & & \\
\hline Transverse and posterior wall & $2.4 \pm 1.4$ & $2.1 \pm 1.1$ & 9 & 5 & 1 \\
\hline T-shape & $3.4 \pm 1.8$ & $2.8 \pm 2.0$ & 9 & 6 & 2 \\
\hline Anterior column and posterior hemitransverse & $3.1 \pm 2.3$ & $2.0 \pm 1.9$ & 6 & 5 & 1 \\
\hline Both column & $3.6 \pm 2.7$ & $2.5 \pm 1.5$ & 11 & 9 & 4 \\
\hline Summary & $2.8 \pm 1.9^{\mathrm{a}, \mathrm{b}}$ & $2.2 \pm 1.4^{b}$ & 80 & 37 & 10 \\
\hline
\end{tabular}

${ }^{a}$ significant difference among different fracture types $(P<0.05)$.

${ }^{b}$ correlation with the quality of fracture reduction $(P<0.05)$. 


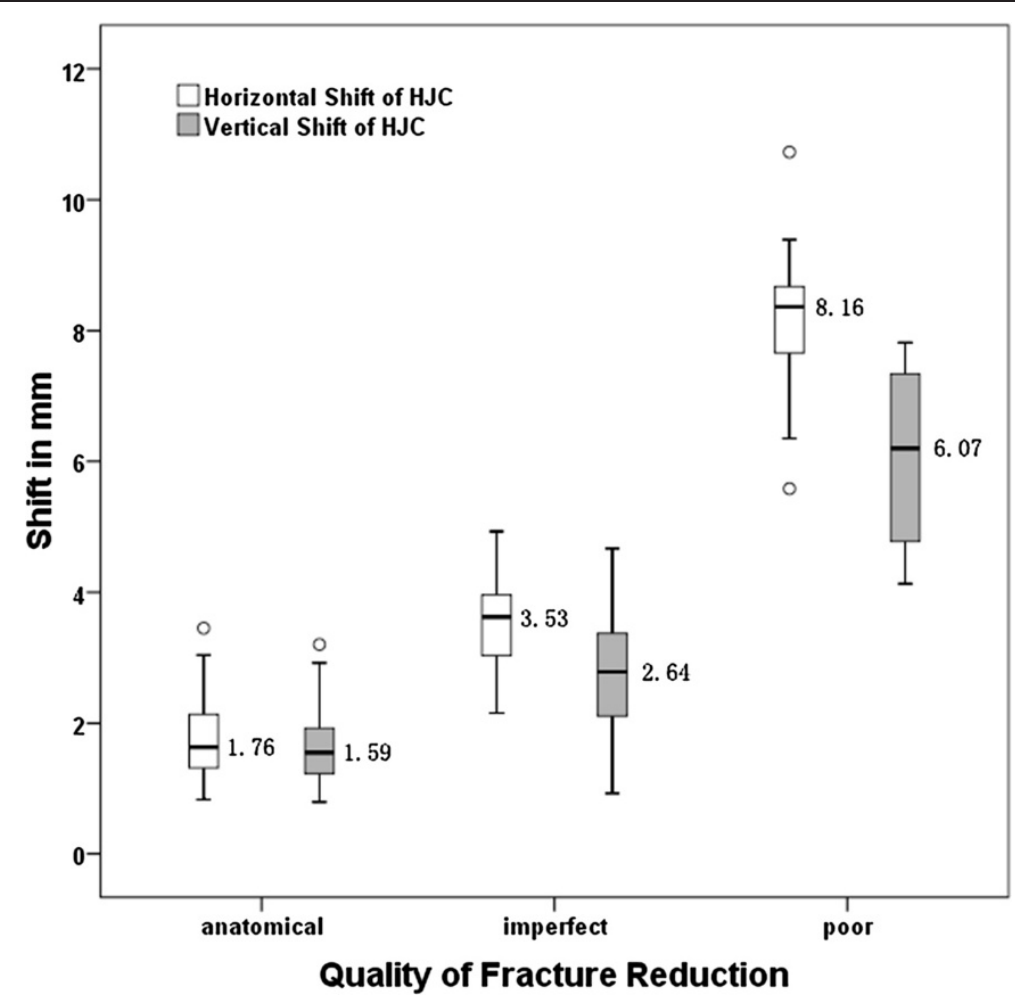

Figure 2 The horizontal and vertical shift of HJC in patients with different quality of fracture reduction. The mean values of the shifts were marked beside the boxplot.

small when an anatomical or imperfect fracture reduction was achieved (Figure 2), the subsequent changes of hip load would probably be acceptable [19]. Considering poorly reduced fractures, which might bear more clinical significance, to what extent will the shift of
HJC affect the hip load changes and the clinical outcomes should be closely analyzed.

Besides the hip load, a shifted HJC may also lead to the changes of surrounding muscle forces in order to balance the moment of body weight. Delp observed a

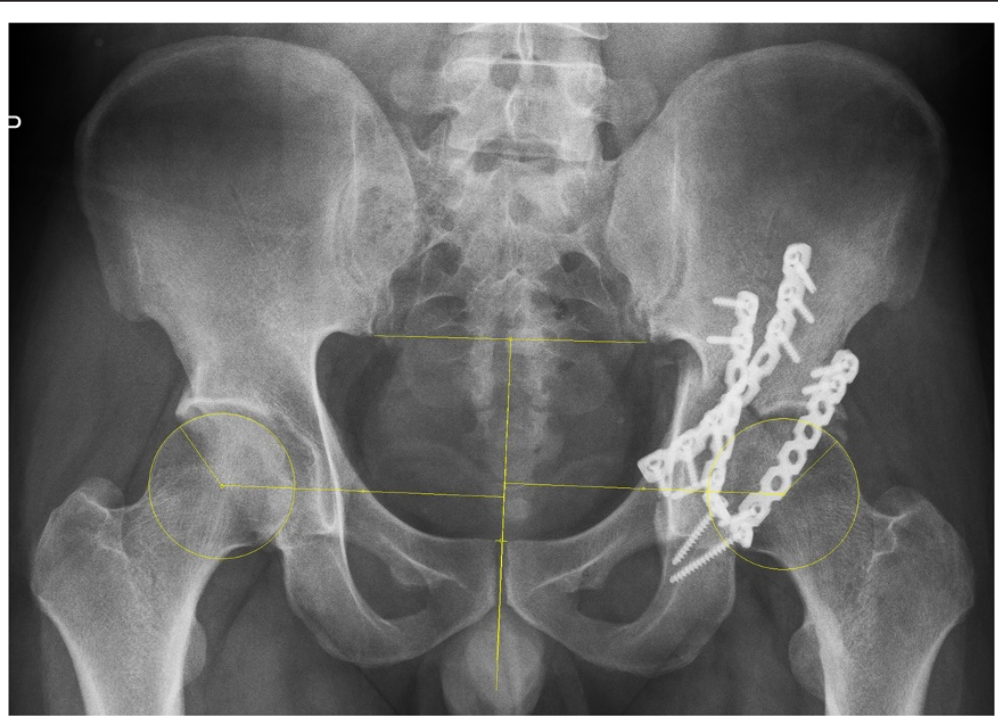

Figure 3 Postoperative shift of HJC in a poorly reduced acetabular fracture. The fracture type is transverse and posterior wall. The horizontal and vertical shifts of HJC were measured to be $6.5 \mathrm{~mm}$ and $5.8 \mathrm{~mm}$ respectively. 
$44 \%$ decrease of abduction force and a $27 \%$ decrease of flexion force following $2 \mathrm{~cm}$ proximal shift of HJC [20]. A $2 \mathrm{~cm}$ medial shift of $\mathrm{HJC}$, in the same study, was testified to reduce $26 \%$ of the adduction force. In our study, again, the mean values of postoperative HJC shifts were relatively small compared to a $2 \mathrm{~cm}$ scale. Therefore the potential contribution of the shifted HJC to the subsequent muscle imbalance and gait changes might be trivial. However, future studies using experimental or computer models would be needed to provide direct evidence for this hypothesis.

Radiographic criteria suggested by Matta are generally used to evaluate the quality of fracture reduction [11]. In our study, an anatomical reduction was achieved in $75.0 \%$ of the elementary fractures and in $53.5 \%$ of the associated fractures, while the rate of poor reduction was $3.6 \%$ and $11.3 \%$ respectively. These were comparable with the results of the other studies [2,21]. An important finding of our study was that the postoperative shifts of HJC were correlated with the quality of fracture reduction. This was reasonable since anatomical reduction would theoretically lead to an ideal restoration of HJC, while a poorly reduced fracture might leave residual displacements of columns and/or walls to hinder the restoration of HJC. Based on this finding, the quality of fracture reduction graded using Matta' criteria might imply the status of HJC restoration. An anatomical fracture reduction, therefore, should be aimed and checked intraoperatively to restore an optimal HJC.

In this study, the horizontal shift of HJC was found to be correlated with the fracture types. This reflected the clinical reality that an associated-type or so-called complex acetabular fracture would lead to an increased duration of surgery, a decreased quality of fracture reduction, and a higher value of horizontal shift of HJC. Specifically, patients with a both-column or T-shape type of fracture presented highest value of horizontal shift of $\mathrm{HJC}$. Meanwhile, the highest rate of poor functional outcome, as reported by Briffa's, was observed in the patients with a posterior column, posterior column and posterior wall, or posterior wall type of fracture [2]. This inconsistency between the radiographic and functional evaluations was also reported by Magill previously [22]. As a potential influencing factor for the horizontal shift of HJC, the displacement of the quadrilateral plate was not analyzed in this study because it's not specifically considered in the Matta's grading system.

Various methods have been reported to determine the anatomical HJC on two-dimensional pelvic radiographs. Anatomical landmarks like teardrops, Shenton's line, Köhler's line, and inter-sacroiliac line were used by different investigators, while the HJC was testified to be most precisely determined referring to the teardrops [23]. However, in our pilot study, the ipsilateral teardrop could only be precisely identified in less than $20 \%$ of the postoperative pelvic radiographs due to fracture disruption or implant obstruction. Therefore we used the contralateral intact acetabulum and femoral head as mirrored template to determine the estimated HJC. Similar methods were reported previously in other studies, showing acceptable accuracy and repeatability $[9,24]$.

This study has a few limitations. First, we only used two-dimensional radiographs to examine the postoperative HJC. Although it's a common practice in hip arthroplasty, the consistence between an HJC identified in anteroposterior radiographs and that located using functional method was questioned recently [25]. CT scan, three-dimensional image analysis or even computer navigation system, if practical, might provide better information considering HJC location and shape changes of the acetabulum in future studies. Second, we didn't evaluate biomechanical alterations or follow-up data of functional outcome caused by the shift of HJC in the current study. Based on our findings, special emphasis will be placed on the patients with poor quality of fracture reduction, to investigate the biomechanical consequence as well as functional changes caused by the shift of HJC in our further studies.

\section{Conclusions}

In conclusion, varying degrees of medial and proximal shifts of HJC were observed in the majority of the acetabular fractures following ORIF. The postoperative restoration of HJC showed significant correlation with the quality of fracture reduction. A perfect fracture reduction should be aimed to achieve appropriate HJC restoration. Further studies are required to address the effects of HJC shift on the biomechanical changes and clinical outcomes of hip joint, especially in poorly reduced acetabular fractures.

\section{Abbreviations}

AP: Anteroposterior; CT: Computed tomography; HJC: Hip joint center; ICC: Interclass correlation coefficient; MRD: Maximum residual displacement; ORIF: Open reduction and internal fixation; THA: Total hip arthroplasty; VA line: Vertical axis of the pelvis; $X$ : Horizontal shift of hip joint center; $Y$ : Vertical shift of hip joint center.

\section{Competing interest}

There's no competing interest. No benefits in any form have been received or will be received from a commercial party related directly or indirectly to the subject of this article.

\section{Authors' contributions}

All authors read and agreed with the contents of the manuscript. HFS and YXC participated in the study design. All the patients included in this study were operated on by JX and YXC. HFS, and JFW performed the radiographic evaluations. HFS was in charge of interpreting the data analysis and drafting the manuscript. JX and YHW assisted in revising the manuscript. All authors read and approved the final manuscript.

\section{Acknowledgements}

This study was supported by the Starting Grant for Young Scientific and Technological Talents (Nanjing Health Bureau, QYK10147), the National 
Natural Science Foundation of China (81271997), and the Fundamental Research Funds for the Central Universities (0214-1434-0129).

Received: 13 July 2013 Accepted: 1 August 2014 Published: 13 August 2014

\section{References}

1. Giannoudis PV, Grotz MR, Papakostidis C, Dinopoulos H: Operative treatment of displaced fractures of the acetabulum. A meta-analysis. J Bone Joint Surg Br 2005, 87(1):2-9.

2. Briffa N, Pearce R, Hill AM, Bircher M: Outcomes of acetabular fracture fixation with ten years' follow-up. J Bone Joint Surg Br 2011, 93(2):229-236.

3. Tornetta P 3rd: Displaced acetabular fractures: indications for operative and nonoperative management. J Am Acad Orthop Surg 2001, 9(1):18-28.

4. Olson SA, Bay BK, Chapman MW, Sharkey NA: Biomechanical consequences of fracture and repair of the posterior wall of the acetabulum. J Bone Joint Surg Am 1995, 77(8):1184-1192.

5. Vrahas MS, Widding KK, Thomas KA: The effects of simulated transverse, anterior column, and posterior column fractures of the acetabulum on the stability of the hip joint. J Bone Joint Surg Am 1999, 81(7):966-974.

6. Della Valle AG, Padgett DE, Salvati EA: Preoperative planning for primary total hip arthroplasty. J Am Acad Orthop Surg 2005, 13(7):455-462.

7. Barrack RL, Burnett SJ: Preoperative planning for revision total hip arthroplasty. J Bone Joint Surg Am 2005, 87(12):2800-2811.

8. Iglic A, Antolic V, Srakar F: Biomechanical analysis of various operative hip joint rotation center shifts. Arch Orthop Trauma Surg 1993, 112(3):124-126.

9. Bicanic G, Delimar D, Delimar M, Pecina M: Influence of the acetabular cup position on hip load during arthroplasty in hip dysplasia. Int Orthop 2009, 33(2):397-402

10. Borrelli I Jr, Ricci WM, Steger-May K, Totty WG, Goldfarb C: Postoperative radiographic assessment of acetabular fractures: a comparison of plain radiographs and CT scans. J Orthop Trauma 2005, 19(5):299-304.

11. Matta JM: Fractures of the acetabulum: accuracy of reduction and clinical results in patients managed operatively within three weeks after the injury. J Bone Joint Surg Am 1996, 78(11):1632-1645.

12. Matta JM, Mehne DK, Roffi R: Fractures of the acetabulum. Early results of a prospective study. Clin Orthop Relat Res 1986, 205:241-250.

13. Tile M, Helfet D, Kellam J, Tile M: Fractures of the pelvis and acetabulum. $3 \mathrm{rd}$ edition. Philadelphia: Lippincott Williams \& Wilkins; 2003.

14. Murphy D, Kaliszer M, Rice J, McElwain JP: Outcome after acetabular fracture. Prognostic factors and their inter-relationships. Injury 2003, 34(7):512-517

15. Mears DC, Velyvis JH, Chang CP: Displaced acetabular fractures managed operatively: indicators of outcome. Clin Orthop Relat Res 2003, 407:173-186.

16. Doehring TC, Rubash HE, Shelley FJ, Schwendeman LJ, Donaldson TK, Navalgund YA: Effect of superior and superolateral relocations of the hip center on hip joint forces. An experimental and analytical analysis. J Arthroplasty 1996, 11(6):693-703.

17. Karachalios T, Hartofilakidis G, Zacharakis N, Tsekoura M: A 12- to 18-year radiographic follow-up study of Charnley low-friction arthroplasty. The role of the center of rotation. Clin Orthop Relat Res 1993, 296:140-147.

18. Delp SL, Wixson RL, Komattu AV, Kocmond JH: How superior placement of the joint center in hip arthroplasty affects the abductor muscles. Clin Orthop Relat Res 1996, 328:137-146.

19. Bayley JC, Christie MJ, Ewald FC, Kelley K: Long-term results of total hip arthroplasty in protrusio acetabuli. J Arthroplasty 1987, 2(4):275-279.

20. Delp SL, Maloney W: Effects of hip center location on the momentgenerating capacity of the muscles. J Biomech 1993, 26(4-5):485-499.

21. Ochs BG, Marintschev I, Hoyer H, Rolauffs B, Culemann U, Pohlemann T, Stuby FM: Changes in the treatment of acetabular fractures over 15 years: Analysis of 1266 cases treated by the German Pelvic Multicentre Study Group (DAO/DGU). Injury 2010, 41(8):839-851.

22. Magill P, McGarry J, Queally JM, Morris SF, McElwain JP: Minimum ten-year follow-up of acetabular fracture fixation from the Irish tertiary referral centre. Injury 2012, 43(4):500-504.
23. Schofer MD, Pressel T, Heyse TJ, Schmitt J, Boudriot U: Radiological determination of the anatomic hip centre from pelvic landmarks. Acta Orthop Belg 2010, 76(4):479-485.

24. Kim DH, Cho SH, Jeong ST, Park HB, Hwang SC, Park JS: Restoration of the center of rotation in revision total hip arthroplasty. J Arthroplasty 2010 25(7):1041-1046.

25. Bouffard V, Begon M, Champagne A, Farhadnia P, Vendittoli PA, Lavigne M, Prince F: Hip joint center localisation: a biomechanical application to hip arthroplasty population. World J Orthop 2012, 3(8):131-136.

doi:10.1186/1471-2474-15-277

Cite this article as: Shi et al:: Radiographic analysis of the restoration of hip joint center following open reduction and internal fixation of acetabular fractures: a retrospective cohort study. BMC Musculoskeletal Disorders 2014 15:277.

\section{Submit your next manuscript to BioMed Central and take full advantage of:}

- Convenient online submission

- Thorough peer review

- No space constraints or color figure charges

- Immediate publication on acceptance

- Inclusion in PubMed, CAS, Scopus and Google Scholar

- Research which is freely available for redistribution 